\title{
1 Structure Determination of Inactive-State GPCRs with a Universal Nanobody
}

3 Michael J. Robertson ${ }^{1,2}$, Feng He ${ }^{1,2}$, Justin G. Meyerowitz ${ }^{1,2,3}$, Alpay B. Seven ${ }^{1,2}$, Ouliana

4 Panova $^{1,2}$, Maria-Claudia Peroto ${ }^{1,2}$, Tao Che $^{4,5}$, Georgios Skiniotis ${ }^{1,2,6}$

6 1Department of Molecular and Cellular Physiology, Stanford University School of 7 Medicine, Stanford, CA, USA

$8{ }^{2}$ Department of Structural Biology, Stanford University School of Medicine, Stanford, CA, 9 USA

10 3Department of Anesthesiology, Perioperative and Pain Medicine, Stanford University 11 School of Medicine, Stanford, CA 94305, USA.

$12{ }^{4}$ Department of Anesthesiology, Washington University School of Medicine, St. Louis, 13 Missouri, 63110, USA.

$14{ }^{5}$ Center for Clinical Pharmacology, University of Health Sciences \& Pharmacy at St. Louis

15 and Washington University School of Medicine, St. Louis, Missouri, 63110, USA.

16 Correspondence: yiorgo@stanford.edu

18 Summary

19 Cryogenic electron microscopy (cryo-EM) has widened the field of structure-based drug

20 discovery by allowing for routine determination of membrane protein structures previously

21 intractable. However, despite representing one of the largest classes of therapeutic

22 targets, most inactive-state G protein-coupled receptors (GPCRs) have remained

23 inaccessible for cryo-EM because their small size and membrane-embedded nature 
24 impedes projection alignment for high-resolution map reconstructions. Here we

25 demonstrate that a camelid single-chain antibody (nanobody) recognizing a grafted

26 intracellular loop can be used to obtain cryo-EM structures of different inactive-state

27 GPCRs at resolutions comparable or better than those obtained by X-ray crystallography.

28 Using this approach, we obtained the structure of human neurotensin 1 receptor (NTSR1)

29 bound to antagonist SR48692, of $\mu$-opioid receptor (MOR) bound to the clinical

30 antagonist alvimopan, as well as the structure of the previously uncharacterized

31 somatostatin receptor 2 (SSTR2) in the apo state; each of these structures yields novel

32 insights into ligand binding and specificity. We expect this rapid, straightforward approach

33 to facilitate the broad structural exploration of GPCR inactive states without the need for

34 extensive engineering and crystallization.

\section{Introduction}

37 The impact of cryo-EM on structure-based drug discovery has been immense,

38 leading to the characterization of a wide variety of new and valuable membrane protein

39 drug targets, including ion channels and G protein-coupled receptors (GPCRs) ${ }^{1}$. As a key

40 requirement for high-resolution cryo-EM map reconstructions, the randomly oriented

41 particle projections must first be correctly aligned at intermediate-to-low resolution, a step

42 that often fails for small proteins due to their limited structural features. For relatively small

43 membrane proteins, the problem is compounded by the presence of a detergent micelle

44 or lipid nanodisc used for solubilization, which further dampens the contrast of protein

45 densities. This poses a major bottleneck for cryo-EM structure determination of GCPRs,

46 which account for roughly $35 \%$ of FDA-approved drugs, with approximately half of those 
47 being agonists that activate the receptor and half antagonists or inverse agonists ${ }^{2}$ that

48 engage the inactive-state receptor, thereby blocking endogenous signaling. Active-state

49 GPCR structures can be determined in complex with heterotrimeric G protein ${ }^{3}$, a $~ 90$ kDa

50 entity that adds significant mass outside the detergent micelle or lipid disc and provides

51 a source of alignment for the transmembrane receptor. However, there is no such

52 generalizable tool for cryo-EM structure determination of inactive-state GPCRs,

53 especially for family A receptors, the largest GCPR subfamily with hundreds of receptors

54 that are typically $\sim 37-50 \mathrm{kDa}$ and lack sizeable extracellular domains for projection

55 alignment. Thus, the application of cryo-EM is severely limited for inactive-state GPCRs,

56 and their structural elucidation mostly relies on extensive engineering and crystallization

57 trials for X-ray studies. This limitation impacts not only mechanistic investigations of

58 GPCRs, but also structure-based drug discovery for the countless therapeutically relevant

59 receptors.

60 Che et al. $^{4}$ recently reported a camelid $\mathrm{VHH}$ domain antibody, referred to as

61 nanobody $6(\mathrm{Nb6})$, that engages the inactive-state intracellular loop 3 (ICL3) of the $\kappa-$

62 opioid receptor (KOR), even when this loop is grafted onto a wide range of receptors,

63 including orphan receptors and family B receptors. This tool was initially developed as a

64 bioluminescence resonance energy transfer (BRET)-based sensor of receptor activation

65 state, an approach that also allows for cost-effective and robust screening of Nb6-binding

66 constructs by employing BRET assays. Using the Nb6 approach, we obtained three-

67 dimensional (3D) map reconstructions of human NTSR1 (hNTSR1) in complex with Nb6

68 bound to the inverse agonist $\mathrm{SR} 48692^{5}$ at a resolution of $2.4 \AA$; the mouse MOR bound 69 to a megabody ${ }^{6}$, an enlarged nanobody construct derived from $\mathrm{Nb6}(\mathrm{Mb} 6)$, in complex 
70 with the antagonist alvimopan ${ }^{7}$ at a resolution of $2.8 \AA$; and the unliganded (apo) structure

71 of human SSTR2 bound to $\mathrm{Nb} 6$ at $3.1 \AA$ resolution. We thus demonstrate that $\mathrm{Nb} 6$ is a

72 general solution for determining high-resolution inactive-state structures by cryo-EM.

73 Besides alleviating the need for extensive construct engineering and successful

74 crystallogenesis, the cryo-EM/Nb6 approach comes with two distinct advantages: first, it

75 does not necessitate the screening and development of receptor-specific nanobodies.

76 Second, the Nb6 BRET-based sensor allows for rapid and efficient screening of

77 constructs and conditions instead of time-consuming and expensive cryo-EM-based

78 screening.

80 Employing Nb6 for cryo-EM structure determination of GPCRs

81 Any fiducial marker that is used for cryo-EM structure determination of a target

82 macromolecule needs to be rigid in its binding and orientation in respect to the structural

83 target. Furthermore, if the marker is particularly small related to the target, it must be

84 positioned such that it provides a distinct feature from various angles to allow

85 unambiguous alignment of the randomly-oriented cryo-EM projections. A crystal structure

86 of $\mathrm{Nb6}$ bound to KOR revealed an interaction at the base of TM5 and TM6, off-center

87 from the major axis of the receptor ${ }^{4}$. We hypothesized that this type of interaction would

88 be an ideal asymmetric fiduciary for cryo-EM particle projection alignment as it would

89 allow for the determination of the rotational orientation of the receptor around its major

90 axis, particularly during initial low-resolution alignments.

91 To evaluate the degree of rigidity of $\mathrm{Nb6}$ when bound to a GPCR, we first

92 performed all-atom molecular dynamics (MD) simulations using the crystal structure of 
93 the KOR-Nb6 complex. Alignment of the MD trajectories on the receptor atoms and

94 calculation of the average root mean squared fluctuation (RMSF) for Nb6 atoms over the

95 final 100 ns of triplicate 500 ns simulations (Fig. 1a) showed that Nb6 is rigidly bound and

96 characterized by little flexibility, with the exception of the distal loops at the base of the

97 nanobody.

$98 \quad$ Encouraged by these computational predictions, we proceeded with employing

$99 \mathrm{Nb6}$ for de novo cryo-EM structure determination of three pharmacologically relevant

100 family A GPCRs. The first, NTSR1, is broadly associated with neurological and

101 gastrointestinal processes ${ }^{8}$. Overexpression of NTSR1 in some tumor types promotes

102 cancer progression and metastasis and is thus associated with poor prognosis ${ }^{9}$,

103 prompting the study and development of NTSR1 inverse agonists for oncology ${ }^{10}$. We also

104 studied MOR, the target of opioids, which are both the most effective treatment for pain

105 but also the therapeutic class underlying the ongoing opioid epidemic in the United

106 States ${ }^{11}$. While current opioid antagonists can treat acute opioid overdose, there is an

107 urgent need for MOR antagonists with improved pharmacokinetic properties ${ }^{11,12}$. Finally,

108 SSTR2 plays a pivotal role in the neuroendocrine system by opposing the release of many

109 hormones, including growth hormone ${ }^{13}$. SSTR agonists are commonly prescribed for

110 neuroendocrine tumors, while SSTR subtype-selective antagonists are in development

111 for diabetes, but the lack of structural information for any somatostatin receptor family

112 member in either active or inactive state has made the development of next-generation

113 SSTR ligands challenging.

114 As described in Che et al., we engineered constructs to enable binding of $\mathrm{Nb6}$ to

115 mouse MOR and human SSTR2 by identifying minimal swaps of ICL3 along with the 
116 cytoplasmic tips of transmembrane helix 5 and 6 (TM5/6) with that of the KOR (Fig. 1c).

117 In the case of the closely related MOR, the two point mutations M264L and K269 ${ }^{6.24} \mathrm{R}$

118 (Ballesteros-Weinstein notation ${ }^{14}$; Fig. 1c) were sufficient to enable Nb6 binding. SSTR2

119 was able to bind Nb6 with the swap of all 15 residues from S238 ${ }^{5.68}$ of TM5 (V256 5.68 of

$120 \mathrm{KOR}$ ) to K252 $2^{6.31}$ of TM6 (R2706.31 of KOR) (Fig. 1c). For human NTSR1 (hNTSR1) we

121 utilized the same swap from KOR T2475.59 to $L 277^{6.38}$ as previously described for rat

122 NTSR14 (rNTSR1; Fig. 1c). BRET assays of agonist-induced Nb6 dissociation confirmed

123 construct functionality, with all three receptors showing dissociation of the nanobody in

124 response to agonist binding (Fig. 1d-f). In all experimental replicates, the BRET data for

125 MOR response to DAMGO appears to be biphasic, consistent with prior nanobody-based

126 MOR activation sensors ${ }^{15}$, where the two-phase response is attributed to distinct plasma

127 membrane and endosomal signaling.

128 High-resolution cryo-EM structure determination was feasible in all cases. Despite

$129 \mathrm{Nb} 6$ being only $12 \mathrm{kDa}$, Nb6, or the equivalent region of $\mathrm{Mb} 6$, is resolved in all three

130 maps, providing a critical stable density outside the detergent micelle surrounding

131 NSTR1, MOR and SSTR2. Notably, the local resolution indications for Nb6 in our

132 GPCR/Nb6 complex cryo-EM maps showed high correlation with the relative root mean

133 square fluctuation (RMSF) values for Nb6 calculated from our MD simulations (Fig. 1b).

134 This observation further demonstrates the strong predictive power of MD simulations in 135 assessing the behavior of fiducial markers for such work. 
139 only available crystal structures were determined in $2021^{16}$ : one with receptor in the apo

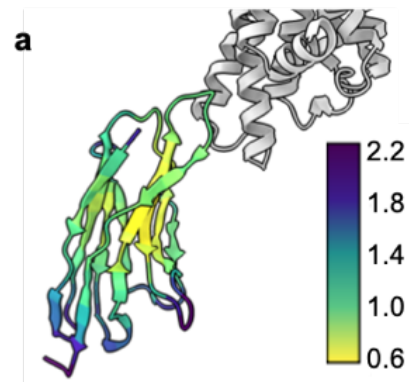

$\operatorname{RMSF}(\AA)$

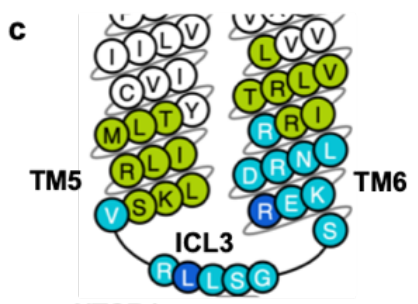

NTSR1 MOR SSTR2

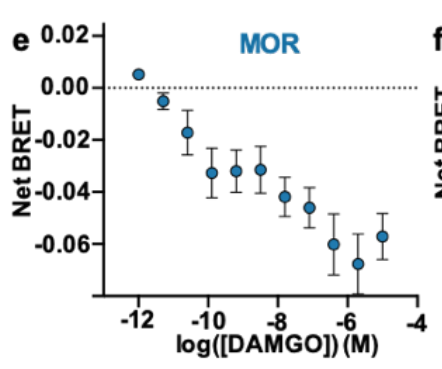

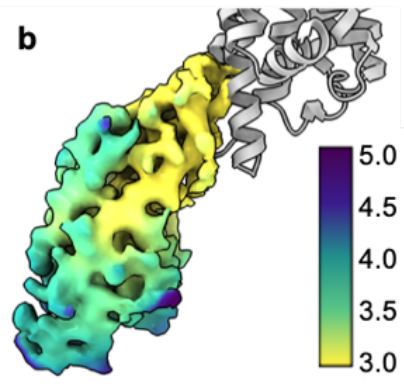

Local Resolution ( $\AA$ )
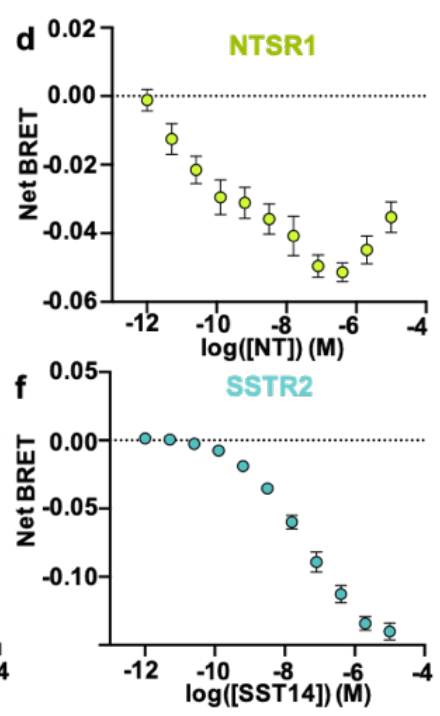

Fig 1. I Construct design and evaluation for Nb6-based GPCR structure determination

a, Root mean squared fluctuations (RMSF) in atomic positions averaged over triplicate molecular dynamics simulations for $\mathrm{Nb6}$ bound to KOR. b, Local resolution plot of $\mathrm{Nb} 6$ from the cryo-EM map of the SSTR2/Nb6 complex. c, Overlay of the three KOR ICL3 constructs used to enable Nb6 binding for MOR (two point mutations), SSTR2 (5.68 to 6.31), and hNTSR1 (5.59 to 6.38). Doseresponse data representing loss of BRET (net BRET) between ReceptorrLuc and Nb6-mVenus for d, NTSR $1_{k}$ and neurotensin (NT), e, $\mathrm{MOR}_{\mathrm{K}}$ and DAMGO, and $\mathbf{f}, \quad$ SSTR2 $_{\mathrm{K}}$ and somatostatin 14 (SST14).

141 state (3.19 $\AA$ ) and two structures with the receptor bound to the inverse agonists SR48692

$142(2.64 \AA)$ or SR142948A (2.92 A), which are small molecules previously in development

143 for treating cancer. Obtaining these structures required extensive engineering to facilitate

144 protein crystallization, with that work employing a construct of rat NTSR1 evolved in

145 bacteria to acquire numerous thermostabilizing mutations and fused in TM7 with a

146 DARPin $^{17}$ protein domain to facilitate crystal packing (rNTSR1-H4) $)^{18}$. To assess and

147 compare the cryo-EM/Nb6 approach with crystallography we obtained the cryo-EM 


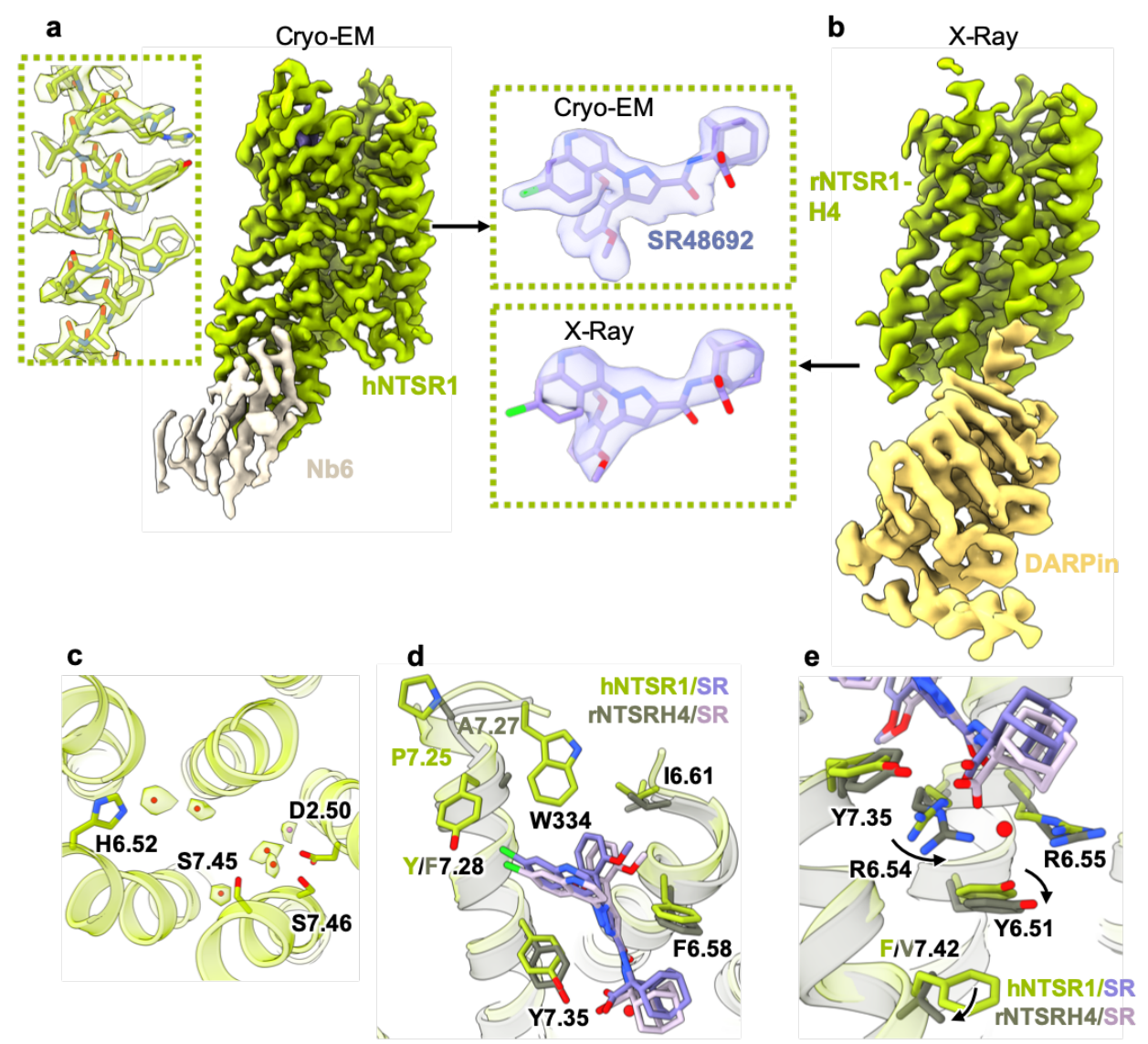

Fig 2. | Comparison of cryo-EM structure of hNTSR1 and crystal structure of rNTSR1-H4

a, Cryo-EM map of hNTSR1 at $2.4 \AA$ global resolution. The arrow points to the cryoEM density and model for inverse agonist SR48692, the inset to the left is the mapmodel fit for TM3 b, 2Fo-Fc crystallography map of rNTSR-H4 at $2.6 \AA$ contoured at $\sigma=1.0$. The arrow points to the Local 2Fo-Fc map around SR48692 contoured at $\sigma=1.25$. c, Water molecules resolved in the core of the hNTSR1 receptor and their associated cryo-EM densities. d, Overlay of the hNTSR cryoEM structure (protein in green, SR48692 in purple) with the rNSTR-H4 crystal structure (protein in gray, SR48692 in pink) highlighting differences in ECL2. e, Overlay of the hNTSR cryoEM structure (protein in green, SR48692 in purple) with the rNSTR-H4 crystal structure (protein in gray, SR48692 in pink) highlighting the residue shift and probable loss of bound water molecule caused by the F7.42V thermostabilizing mutation

148 structure of hNTSR1/Nb6 complex bound to SR48692 (Fig. 2a, Extended Data Fig. 2).

149 Notably, the cryo-EM structure was resolved at a global resolution of $2.4 \AA$, both a higher

150 nominal resolution than the crystal structure and with significantly better-resolved

151 features, including the density corresponding to the ligand (Fig. 2a, b insets, Extended 
152 Data Fig. 8a). This resolution range also reveals densities corresponding to extensive

153 hydration throughout the receptor in the cryo-EM map (Fig. 2c, Extended Data Fig. 3a).

154 The construct for hNTSR1 was minimally modified beyond the KOR ICL3 swap,

155 with only $\mathrm{N}$ - and $\mathrm{C}$ - termini truncations in regions not resolved in typical family A structures

156 and a single $\mathrm{A} 85^{1.54} \mathrm{~L}$ mutation to increase expression ${ }^{19}$. While the general ligand pose is

157 very similar between the two structures, the cryo-EM structure reveals additional

158 interactions not observed in the rNTSR-H4 crystal structure. First, the remodeling of the

159 TM7-ECL3 region allows W334 in ECL3 to be resolved in the hNTSR1 structure loosely

160 capping the top of the hydrophobic chloro-naphthyl and dimethoxy-phenyl moieties of

161 SR48692 (Fig. 2c). This change may be due to several amino acid sequence differences

162 at the top of TM7, most prominently P336 ${ }^{7.25}$ in hNTSR1 versus T341 $1^{7.25}$ in rNTSR1-H4,

163 with the proline inducing a sharp turn between the top of TM7 and ECL3 in hNTSR1. In

164 addition, the rearrangement of R327 $7^{6.54}$ in rNTSR-H4 (R322 ${ }^{6.54}$ in hNTSR1) appears to

165 disrupt a water-mediated interaction between R327 $7^{6.54}, R_{328^{6.55}}$ (R323 $3^{6.55}$ in hNTSR1),

166 and the carboxylate moiety of SR48692 observed in the hNTSR1 structure (Fig. 2d). This

167 R327 $7^{6.54}$ rearrangement likely stems from the $\mathrm{F} 358^{7.42} \mathrm{~V}$ thermostabilizing mutation in

168 rNTSR-H4, allowing a downward relaxation of Y3246.51 (Y3196.51 in hNTSR1) and

169 R327 ${ }^{6.54}$. Notable differences also exist in the TM helices between the two structures;

170 TM1 is shifted $3.4 \AA$ further outward from the core of the receptor in the crystal structure

171 (Extended Data Fig. 3b), while the top of TM2 and ECL1 are out of register by one residue.

172 While this may be due to the differences in experimental constructs, the observed

173 discrepancy may also result from the fact that even at $\sigma=1$ the 2 Fo-Fc map of the crystal

174 structure lacks sidechain information in the ECL1 region, rendering modeling ambiguous 
175 (Extended Data Fig. 3c,d). Overall, the lack of thermostabilizing mutations and DARPin

176 in TM7 restores a more native-like inactive state as suggested by the conformation of key

177 amino acids. For example, Y364 ${ }^{7.53}$ of the hNTSR1 NPxxY motif (Y369 ${ }^{7.53}$ in rNSTR1)

178 occupies a position and rotamer more typical of an inactive state family A GPCR 179 (Extended Data Fig. 3e).

181 Structure of MOR

In parallel, we obtained a cryo-EM structure of the MOR bound to the non-blood

183 brain barrier penetrant, MOR selective inverse agonist alvimopan, an FDA-approved drug

184 for reversing opioid-induced gastrointestinal (GI) symptoms in hospital settings. In the

185 case of NTSR1/Nb6, vitrifying the sample in a very thin buffer layer on holey gold grids ${ }^{20}$

186 was necessary, not only for high resolution but also to achieve proper alignment of particle

187 projections. To assess an alternative approach, we used Nb6 to develop a megabody,

188 termed 'Mb6', a fusion of a nanobody and a scaffold protein of either 45 or $86 \mathrm{kDa}$ that

189 can serve as a larger fiducial marker, based on the work of Uchański et al. ${ }^{6}$. In that work,

190 the Mb designs employed for $\mathrm{GABA}_{\mathrm{A}}$ and WbaP produced cryo-EM maps that could not

191 resolve well past the nanobody portion, presumably due to flexibility. We thus opted

192 instead for using the c7HopQA12 design, which incorporates a twisted linker between the

193 nanobody and the $45 \mathrm{kDa} H o p Q$ scaffold to increase their contact and, ideally, rigidity.

194 However, our MD simulations suggested that even this design was characterized by 195 substantial flexibility between the $\mathrm{Nb}$ and scaffold (Extended Data Fig. 5a). In agreement

196 with this result, our cryo-EM 2D class averages of the MOR-Mb6 complex showed that

197 only the Nb6-receptor portion is well resolved, with the scaffold averaging out (Extended 
198 Data Fig. 4b). Nevertheless, leveraging the ordered Nb6 portion of Mb6 in our cryo-EM

199 data, we obtained a $2.8 \AA$ A cryo-EM map (Fig. 3a, Extended Data Fig. 4) that provided an

200 unambiguous density for the ligand and receptor (Fig. 3c, Extended Data Fig. 8b). This

201 level of detail is quite comparable to the $2.8 \AA$ crystal structure of MOR (Fig. 3b, d),

202 although the cryoEM map further resolves several probable water molecules in the ligand

203 binding site, including one that bridges interactions between the carboxylate of alvimopan

204 and the receptor (Extended Data Fig. 5b).

205 Comparing the binding poses of the MOR-selective alvimopan with that of the

206 structurally similar ligand JDTic, which is selective for KOR, reveals a striking difference.

207 Despite their similar scaffolds, the phenol-piperidine moieties of alvimopan and JDTic are

208 in completely different positions and orientations, nearly flipped in the binding pocket.

209 Aligning our structure with KOR-JDTic $\left(\mathrm{PDB}: 4 \mathrm{DJH}^{21}\right)$ reveals that JDTic would clash with

$210 \mathrm{Y} 326^{7.43}$ and Q124 2.60 in MOR, while alvimopan would clash with W124 and I294.65 in

211 KOR (I294 ${ }^{6.55}$ of KOR is substituted by a repositioned valine in MOR). In addition to the

212 steric confines of the binding pocket, charge differences also appear to contribute to the

213 differences in binding pose between JDTIC and alvimopan. The nitrogen of the

214 tetrahydroisoquinoline ring system of JDTic is likely also protonated or perhaps

215 protonated instead of the piperidine, and thus forms salt bridge interactions with D138 $8^{3.32}$

216 (D147 3.32 in MOR), just as the protonated piperidine of alvimopan does in MOR. Of note,

217 alvimopan shares similarities to fentanyl and its analogues, including a central piperidine

218 ring. Computational docking of carfentanil|22, a potent MOR agonist, in the active-state

219 structure of the receptor ${ }^{3}$ suggests a pose similar to alvimopan, but with the additional

220 phenylamine moiety on the piperidine ring positioned to bind into the pocket occupied by 
221 the toggle switch tryptophan W2936.48 (Extended Data Fig. 5a). How a MOR ligand 222 engages this region is often the main determining factor for its agonism versus

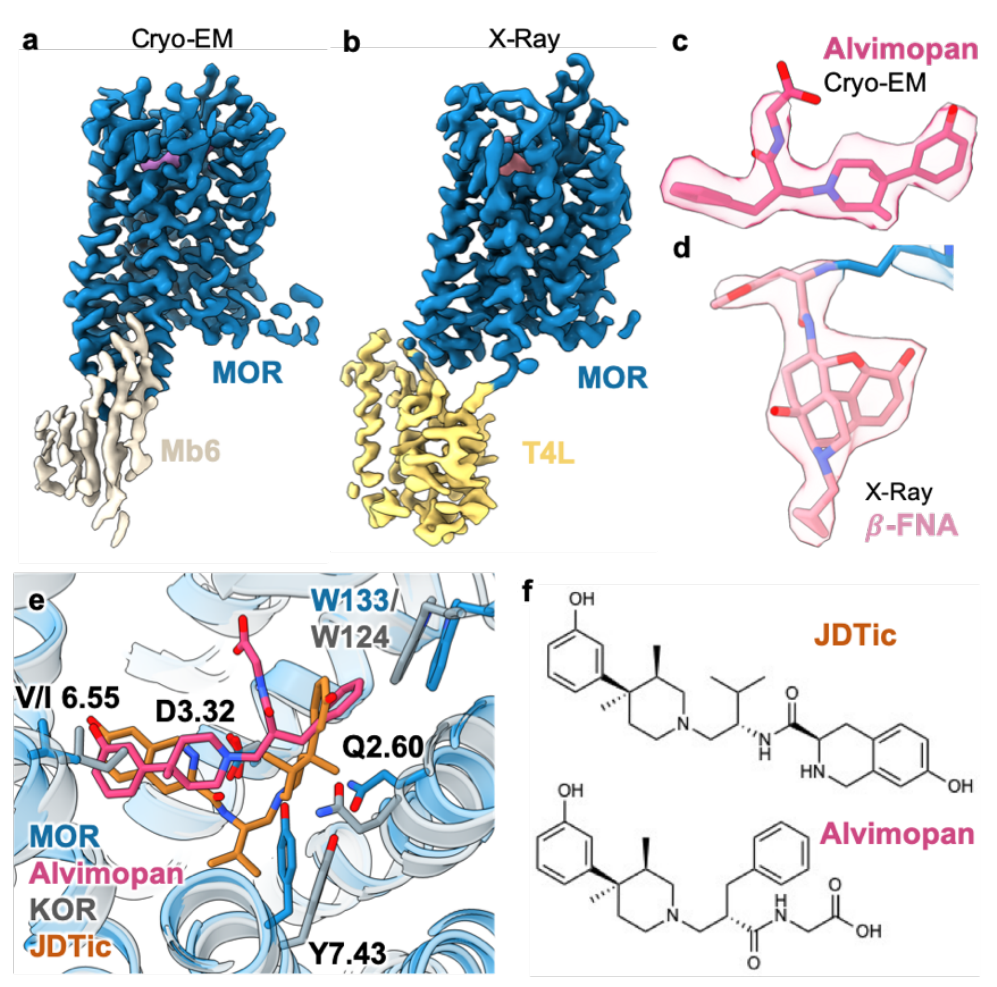

Fig 3. | Comparison of cryo-EM structure of alvimopan-bound MOR with crystal structures of MOR/ $\beta$-FNA and KOR/JDTic a, Cryo-EM map of MOR at $2.8 \AA$ global resolution. b, 2Fo-Fc crystallography map of MOR at $2.8 \AA$ contoured at $\sigma=2.0$. c, Cryo-EM density and model for inverse agonist alvimopan. d, Local 2Fo-Fc map around $\beta$-FNA contoured at $\sigma=1.5$. e, overlay of the alvimopan pose in the MOR cryo-EM structure (protein in blue, alvimopan in magenta) with the JDTic pose in the KOR crystal structure (protein in grey, JDTic in orange). $f$, Comparison of JDTic and alvimopan chemical structures highlighting similar phenol-piperidine scaffold.

223 antagonism, and we hypothesize that this phenyl moiety of fentanyl and its derivatives is

224 responsible for conversion of the piperidine scaffold to an agonist.

\section{Structure of SSTR2}

To test the cryo-EM/Nb6 approach on a receptor without prior structural 229 characterization we chose SSTR2, a member of the SSTR subfamily for which no 230 structures have been determined. Despite the absence of a stabilizing inverse agonist or 231 any other ligand and the lack of an energy filter in the microscope used for cryo-EM data 232 acquisition (typically beneficial for data quality when imaging small proteins), we were 233 able to obtain a $3.1 \AA$ map of SSTR2/Nb6 with well-resolved features (Fig. 4a, Extended 
234 Data Fig. 6, Extended Data Fig. 8c). Together with two active-state structures of SSTR2

235 bound to two different agonists, the endogenous 14-mer SST14 and the synthetic 8-mer

236 octreotide (detailed in our accompanying manuscript ${ }^{23}$ ), the inactive-state SSTR2

237 structure enabled us to characterize several aspects of receptor activation and ligand

238 recognition. Perhaps the most interesting feature of SSTR2 is the conformational flexibility

239 of ECL2; while in a well-defined position in the apo state structure, ECL2 is pushed to a

240 more open conformation when bound to the endogenous agonist SST14, but folds down

241 over the top of the receptor in the octreotide-bound state (Fig. 4b). As a consequence of

242 the ECL2 plasticity, different residues are engaged in binding the pan-SSTR agonist

243 SST14 versus the SSTR2-selective octreotide. This is an important factor to SSTR

244 subtype selectivity, which is explored further in the accompanying study ${ }^{23}$.

245 Very few structures have been obtained to date for family A GPCRs in the

246 unliganded, apo state, primarily due to the general need for a stabilizing ligand to obtain

247 a crystal structure. Experimentally-derived apo structures provide an opportunity to

248 evaluate predicted structures from the recently published deep-learning based tools,

249 AlphaFold $2^{24}$ and RoseTTAfold ${ }^{25}$. Notably, while both software performed well in

250 predicting the overall structure of SSTR2, key details of the ligand binding site were in

251 disagreement with the experimental structure, particularly in residues D122 ${ }^{3.32}, \mathrm{~F} 92^{2.53}$,

252 and $Y 302^{7.43}$ (Fig. 4c), which are involved in ligand binding and activation, as detailed in

253 the companion active-state SSTR2 manuscript ${ }^{23}$. Although a more systematic

254 comparison is needed, this observation underscores both the merits but also the potential

255 limitations of homology model-based approaches. Differences in side chain positioning, 
256 especially within a ligand binding site, can throw off structure-based drug discovery efforts

257 and other pursuits in which high-accuracy coordinates are required.

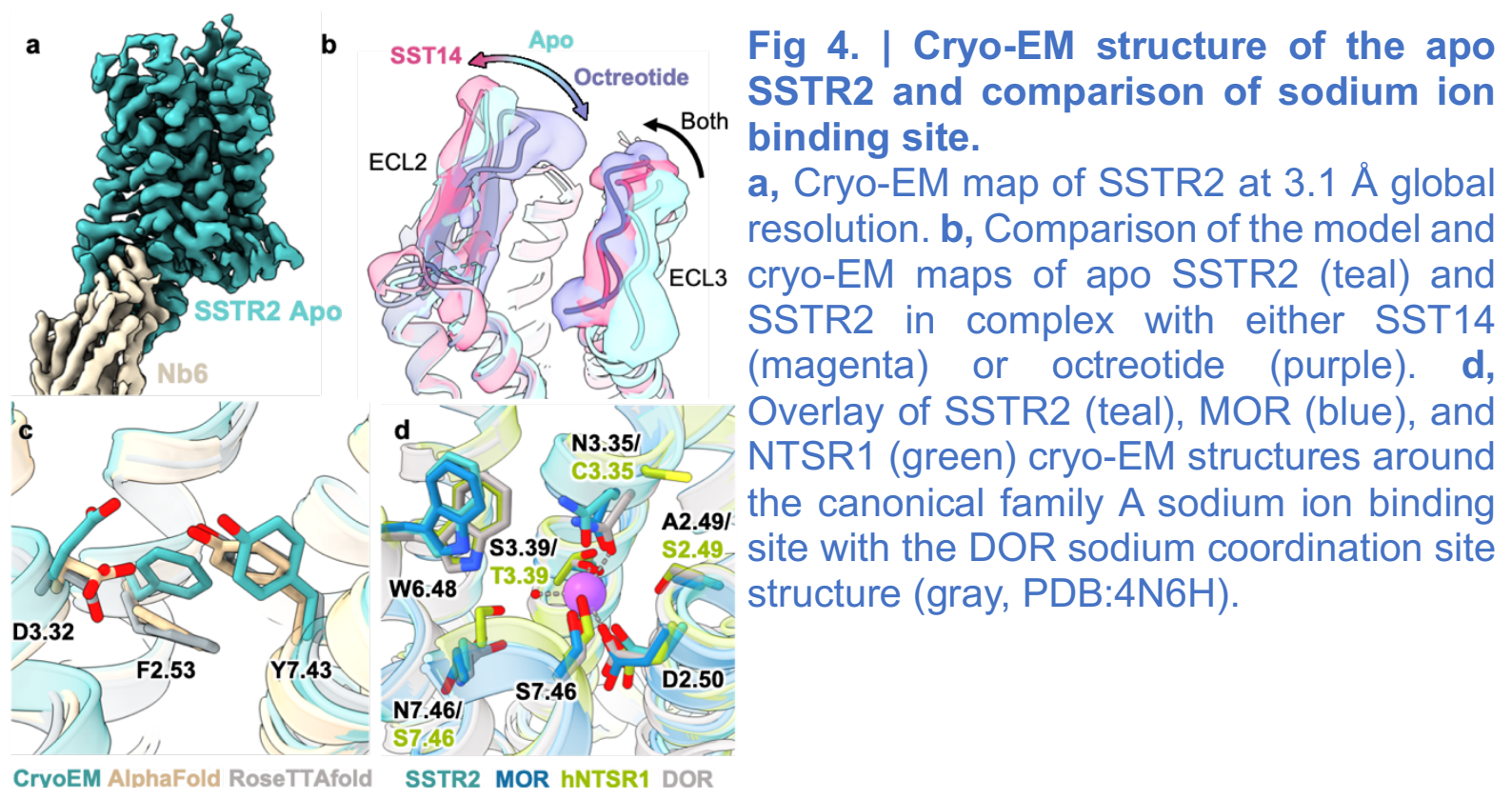

260 Differential sodium accommodation by family A GPCRs

Our near-native inactive-state cryo-EM maps provide the opportunity to examine

262 the allosteric sodium binding site found deep in the core of many family A receptors. All

263 three of the receptors studied in this work have experimentally demonstrated negative

264 allosteric modulation by sodium ${ }^{26-28}$. In both MOR and SSTR2 the identities of the

265 residues in the sodium binding site are the same as the $\delta$-opioid receptor (DOR), for which

266 there is a high-resolution crystal structure $(1.8 \AA)$ with a well-defined sodium site for

267 comparison ${ }^{29}$ (Fig. 4c). The geometries of the MOR, SSTR2, and DOR sites are very

268 similar, with the exception of a slight rearrangement of $W^{6.48}$ in the case of both MOR

269 (W293 ${ }^{6.48}$ ) and SSTR2 (W269 ${ }^{6.48}$ ), and N125 3.35 in SSTR2. In contrast to SSTR2 and

270 MOR, hNTSR1 has four amino acid differences in the sodium binding site, namely 
$271 \mathrm{~T} 155^{3.39}$ (in place of $\mathrm{S}^{3.39}$ ), $\mathrm{S} 357^{7.46}$ (in place of $\mathrm{N}^{7.46}$ ), $\mathrm{S} 111^{2.49}$ (in place of $\mathrm{A}^{2.49}$ ), and

$272 \mathrm{C} 151^{3.35}$ (in place of $\mathrm{N}^{3.35}$ ). In the DOR structure, both $\mathrm{S} 135^{3.39}$ and $\mathrm{N} 131^{3.35}$ directly

273 interact with the sodium .

274 The three maps we determined resolve features consistent with water and/or ions

275 in the canonical sodium binding region near D2.50, although only NTSR1 has sufficiently

276 high resolution in this particular region to facilitate unambiguous ion modeling (Extended

277 Data Fig. 7a-c). The probable ion sites of SSTR2 and MOR match relatively well to those

278 observed in DOR. In the hNTSR1 map, we observe a density with close proximity to 279 oxygen atoms of $\mathrm{T} 155^{3.39}, \mathrm{~S} 111^{2.49}, \mathrm{D} 112^{2.50}$ (Extended Data Fig. 7a). Even though

280 precise identification of water versus ion is challenging in cryo-EM maps, we expect that

281 this feature corresponds to the sodium, shifted by $1.8 \AA$ compared to the sodium in DOR

282 (Fig. 4d, Extended Data Fig. 7d, e). As other receptors with resolved sodium ion sites

283 have cysteine or other hydrophobic residues in place of $\mathrm{N}^{3.35}, \mathrm{~S} 111^{2.49}$ is likely responsible

284 for the shift in sodium position in NTSR1. Overall, we find substantial similarity between

285 the sodium binding sites of inactive state GPCRs from cryo-EM and crystallography.

\section{Discussion}

288 Here, we present inactive-state structures of three family A GPCRs, all obtained 289 by cryo-EM at resolutions as high as $2.4 \AA$ by employing minimal engineering and a 290 universal nanobody for projection alignment. By using molecular dynamics simulations to 291 assess fiducial markers for use in cryo-EM structure determination, we could accurately 292 predict that Nb6 is sufficiently rigid for cryo-EM particle alignment, while Mb6 is too flexible 293 for resolution beyond the nanobody portion. Each of the obtained cryo-EM structures 
294 yields new insights into ligand recognition, with resolution that is as good or better than

295 that of analogous structures produced by x-ray crystallography. In contrast to active state

296 GPCR-G protein complexes, where the highest-resolution portion of the map is on the

297 larger $\mathrm{G}$ protein due to the center of alignment, the inactive-state Nb6 GPCR structures

298 display their highest resolution at the receptor core, facilitating accurate modeling of

299 ligands and their surrounding hydration. This generalizable approach opens this broad

300 family of drug targets to rapid, crystallization-free structure determination, with

301 biochemical stability of the receptor and nanobody binding being the only limitations. As

302 such, we anticipate Nb6 to be a broadly applicable approach to determining inactive state

303 structures of near-native GPCRs, further propelling drug discovery in this class of

304 receptors.

305

306 Author Contributions

307 M.J.R. cloned constructs, expressed and purified proteins, processed EM data, built

308 models, and ran/analyzed molecular dynamics simulations. F.H. cloned constructs and

309 expressed proteins. J.M. performed BRET assays. A.S. prepared cryo-EM samples and

310 collected cryo-EM data. M.C. expressed proteins and purified nanobody. O.P. prepared

311 cryo-EM samples and collected cryo-EM data. T.C. provided constructs for nanobody

312 expression and BRET assays. M.J.R. and G.S. wrote the manuscript with input from F.H.,

313 J.M., O.P., and T.C.. G.S. supervised the project.

314

315 Data Availability 
316 All data generated or analyzed in this study are included in this article and the

317 Supplementary Information. The cryo-EM density maps and corresponding coordinates

318 have been deposited in the Electron Microscopy Data Bank (EMDB) and the Protein Data

319 Bank (PDB), respectively, under the following accession codes: Raw data has been

320 deposited in EMPIAR under the following accession codes:

321

322 Competing Interests

323 The authors declare no competing interests.

324

325 Acknowledgements

326 We thank the Kobilka lab for providing plasmids of NTSR1 and MOR. Cryo-EM data

327 were collected at the Stanford cryo-EM center (cEMc) with support from E. Montabana.

328 This work was supported, in part, by the Mathers Foundation (G.S.), training grant

329 T32GM089626 (J.G.M.), and used the Extreme Science and Engineering Discovery

330 Environment (XSEDE) $)^{30}$ resource comet-gpu through sdsc-comet allocation TG-

331 MCB190153 (G.S.), which is supported by National Science Foundation grant number

$332 \mathrm{ACl}-1548562$.

333

334 Methods

335

336 Cloning 
337 The cDNA for SSTR2 was obtained from Horizon Discovery and cloned into a pFastBac

338 vector containing an $\mathrm{N}$-terminal haemagglutinin $(\mathrm{HA})$ signal sequence followed by a FLAG

339 epitope (DYKDDDD) and a C-terminal C3 protease cleavage site followed by enhanced

340 green fluorescent protein (eGFP) and a hexahistidine (His6) tag using Gibson cloning.

341 Constructs for hNTSR1 and MOR were obtained from the lab of Brian Kobilka and have

342 been described previously ${ }^{3,19}$. Briefly, each is cloned in pFastBac with an N-terminal

343 haemagglutinin (HA) signal sequence followed by a Flag epitope (DYKDDDD) and a C-

344 terminal HRV 3 C protease cleavage site followed by a hexahistidine (His6) tag. In the

345 case of hNTSR1, the His6 tag is preceded by enhanced green fluorescent protein (eGFP).

346 For MOR, two point mutations (M264L and K269R) were introduced with site-directed

347 mutagenesis; PCR reactions were conducted with Q5 polymerase with the following pair

348 of primers: 5'- GGCTCCCGCGAAAAGGACAGGAACCTGCGC -3'; 5'-

349 CGACAGCAGGCGGACACTCTTGAG

$350-3^{\prime}$. For hNTSR1, residues between T247 $7^{5.59}$ to $L 277^{6.3}$ of kappa opioid were substituted

351 with site-directed mutagenesis in three steps, with the following three primer pairs: 5'-

352 ATCACACGCCTGGTTCTGGCAGTGGTCATCGCCT

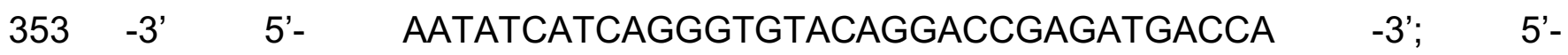

354 GGAATCTGCGCCGAATCACACGCCTGGTTCT

355 ACAGATTTCAGCCGCAATATCATCAGGGTGTACAG-3'; 5'-

356 GCCGCTCAGCAGGCGCACAGATTTCAGCCGCA 5'-

357 AGCCGCGAAAAGGATCGGAATCTGCGCCGAAT -3'. For SSTR2, residues between

358 S238 and K252 were replaced with those of kappa opioid in two steps with site-directed 
mutagenesis

with

the

following

primer

pair:

5'-

360 GAGCGGCAGCTCTAAGAGGAAGAAGTCTGAGAA

361 -3' 5'- AGCAGGCGCACGGACTTCACCTTGATGATAATGAAC

362 -3'; 5'- CGGAATCTGCGCAAGGTCACCCGAATGGTG

363 -3', 5'- ATCCTTTTCGCGGCTGCCGCTCAGCA

$364-3^{\prime}$

365 Biolumescence resonance energy transfer (BRET) assays

366 BRET assays were performed and analyzed as previously described ${ }^{4}$ with the following

367 modifications: HEK-293S cells grown in FreeStyle 293 suspension media (Thermo

368 Fisher) were transfected at a density of 1 million cells $/ \mathrm{mL}$ in $2 \mathrm{~mL}$ volume using $600 \mathrm{ng}$

369 total DNA at 1:1 ratio of Receptor-rLuc:Nb6-mVenus and a DNA:PEI ratio of 1:5,

370 and incubated in a 24 deep well plate at $220 \mathrm{rpm}, 37^{\circ} \mathrm{C}$ for 48 hours. Cells were harvested

371 by centrifugation, washed with Hank's Balanced Salt Solution (HBSS) without

372 Calcium/Magnesium (Gibco), and resuspended in assay buffer (HBSS with $20 \mathrm{mM}$

373 HEPES $\mathrm{pH}$ 7.45) with $1 \mu \mathrm{g} / \mathrm{mL}$ freshly prepared coelenterazine $\mathrm{h}$ (Promega). Cells were

374 plated in white-walled, white-bottom 96 well plates (Costar) in a volume of $60 \mu \mathrm{l} / \mathrm{well}$ and

37560,000 cells/well. Ligands were prepared in drug buffer (assay buffer with $0.1 \%$ BSA, 6

$376 \mathrm{mM} \mathrm{CaCl}_{2}, 6 \mathrm{mM} \mathrm{MgCl}_{2}$ ), and added at a 1:2 ratio of drug:cell suspension. Ten minutes

377 after the addition of ligand, plates were read using a SpectraMax iD5 plate reader using

$378485 \mathrm{~nm}$ and $535 \mathrm{~nm}$ emission filters with a one-second integration time per well. The

379 computed BRET ratios (mVenus/RLuc emission) were normalized to ligand-free control

380 (Net BRET) prior to further analysis. 


\section{Expression and Purification of $\mathrm{Nb6}$ and $\mathrm{Mb6}$}

383 Wk6 E. coli (ATCC) were transformed via heat shock with Nb6 plasmid ${ }^{4}$ and two $5 \mathrm{~mL}$

384 starter cultures were grown overnight in LB supplemented with $100 \mu \mathrm{g} / \mathrm{ml}$ ampicillin. $2 \mathrm{~L}$

385 of terrific broth were supplemented with $100 \mu \mathrm{g} / \mathrm{ml}$ ampicillin, inoculated with starter

386 cultures, and grown at $37^{\circ} \mathrm{C}$ with shaking until $\mathrm{OD}_{600}=0.7$. Expression was then induced

387 with $1 \mathrm{mM}$ IPTG and expression was allowed to proceed at $28^{\circ} \mathrm{C}$ overnight. Pellets were

388 harvested via centrifugation and washed once with phosphate-buffered saline before

389 snap freezing in liquid nitrogen. Pellets containing Nb6 were thawed and resuspended in

$39050 \mathrm{mM}$ Tris pH 8.0, $0.5 \mathrm{mM}$ EDTA, $20 \% \mathrm{w} / \mathrm{v}$ sucrose (TES) buffer at $15 \mathrm{~mL} / 1$ liter pellet

391 and supplemented with protease inhibitor cocktail and shaken in an orbital shaker for 1

392 hour at $4^{\circ} \mathrm{C}$. An additional $30 \mathrm{~mL}$ per 1 liter pellet of TES diluted 1:4 with water was added

393 and placed in an orbital shaker for an additional 45 minutes at $4^{\circ} \mathrm{C}$. Cell debris was

394 removed by ultracentrifugation at $100,000 \times$ g for 30 minutes. The supernatant was filtered

395 with a 45 micron filter, supplemented with $20 \mathrm{mM}$ imidazole, and loaded over a gravity $\mathrm{Ni}$ -

396 NTA column at $4^{\circ} \mathrm{C}$. The column was washed with $10 \mathrm{CV}$ of a buffer containing $250 \mathrm{mM}$

$397 \mathrm{NaCl}, 50 \mathrm{mM}$ Tris $\mathrm{pH}$ 7.5, $10 \mathrm{mM}$ imidazole, and then eluted with buffer containing 250

$398 \mathrm{mM}$ imidazole. Nanobody was concentrated and applied to size exclusion

399 chromatography with a buffer containing $250 \mathrm{mM} \mathrm{NaCl}$ and $50 \mathrm{mM}$ Tris $\mathrm{pH}$ 7.5.

400 Monomeric fractions were pooled, concentrated, supplemented with $10 \%$ glycerol, and

401 snap frozen in liquid nitrogen for later use. Expression and purification of Mb6 was

402 performed identically to Nb6.

403

404 Expression and Purification of Receptor/Nb6 Complexes 
405 All receptors were expressed in Sf9 insect cells (Expression Systems) infected at a 406 density of 3-4 million cells/ml. At 48 hours post-infection, cells were collected with

407 centrifugation, washed with phosphate-buffered saline containing $10 \mu \mathrm{M}$ antagonist 408 (when used), and pellets were snap frozen in liquid nitrogen for purification. A general 409 protocol was used for producing receptor/Nb6 complexes (Extended Data Fig. 1), with 410 some slight deviations. Sf9 pellets containing receptor were lysed in hypotonic buffer 411 containing $20 \mathrm{mM}$ HEPES pH 7.5, $5 \mathrm{mM} \mathrm{MgCl}_{2}$, protease inhibitor cocktail, benzonase, $41210 \mu \mathrm{M}$ antagonist (when used), and $2 \mathrm{mg} / \mathrm{ml}$ iodoacetamide and gently stirred for an hour

413 at $4^{\circ} \mathrm{C}$. Membranes were harvested with ultracentrifugation at $100,000 \mathrm{xg}$, supernatant 414 was discarded, and membranes were resuspended in solubilization buffer containing 250 $415 \mathrm{mM} \mathrm{NaCl}, 20 \mathrm{mM}$ HEPES $\mathrm{pH}$ 7.5, $1 \mathrm{mM} \mathrm{MgCl}_{2}$, protease inhibitor cocktail, $10 \mu \mathrm{M}$ 416 antagonist (when used), and $2 \mathrm{mg} / \mathrm{ml}$ iodoacetamide; then drip frozen into liquid nitrogen 417 and stored at $-80^{\circ} \mathrm{C}$. Resuspended membranes were thawed and detergent was added 418 dropwise while stirring at $4^{\circ} \mathrm{C}$ to a final concentration of $1 \%$ LMNG/0.1\% CHS/0.1\% 419 Cholate. After 3 hours, insoluble debris was removed with ultracentrifugation at 100,000 420 g. Solubilized receptor was supplemented with $20 \mathrm{mM}$ imidazole and gravity loaded over 421 a Ni-NTA resin column. Columns were washed with 10 column volumes of buffer 422 containing $250 \mathrm{mM} \mathrm{NaCl}, 20 \mathrm{mM}$ HEPES pH 7.5, $10 \mu \mathrm{M}$ antagonist (when used), $20 \mathrm{mM}$ 423 imidazole, and $0.1 \%$ LMNG/0.01\% CHS and protein was eluted in buffer containing 250 $424 \mathrm{mM} \mathrm{NaCl}, 20 \mathrm{mM}$ HEPES pH 7.5, $10 \mu \mathrm{M}$ antagonist (when used), $250 \mathrm{mM}$ imidazole, and $4250.01 \%$ LMNG/0.001\% CHS. Receptor was then supplemented with $5 \mathrm{mM} \mathrm{CaCl}_{2}$ and 426 loaded onto M1 flag resin; washed with 5 column volumes of $250 \mathrm{mM} \mathrm{NaCl}, 20 \mathrm{mM}$ 427 HEPES pH 7.5, $10 \mu \mathrm{M}$ antagonist (when used), $2 \mathrm{mM} \mathrm{CaCl}$, and 0.1\% LMNG/0.01\% 
428 CHS; and eluted with buffer containing $150 \mathrm{mM} \mathrm{NaCl}, 20 \mathrm{mM}$ HEPES pH 7.5, $10 \mu \mathrm{M}$

429 antagonist (when used), $1 \mathrm{mM}$ EDTA, $0.2 \mathrm{mg} / \mathrm{ml}$ FLAG peptide, and 0.1\% LMNG/0.01\%

430 CHS. Purified receptor was incubated on ice overnight with HRV 3C protease and purified

$431 \mathrm{Nb6}$ was added at a 2:1 molar ratio. Receptor/Nb6 complex was concentrated in a 50

$432 \mathrm{kDa}$ molecular weight cutoff spin concentrator and subjected to SEC chromatography

433 with an ENrich 650 column (Bio-Rad) and a buffer containing $150 \mathrm{mM} \mathrm{NaCl}, 20 \mathrm{mM}$

434 HEPES pH 7.5, 0.001\% LMNG, 0.00033\% GDN, and 0.0001\% CHS. Fractions containing

435 monomeric receptor/Nb6 complex were pooled for cryo-EM sample preparation. This

436 protocol was identical for SSTR2 and NTSR1 with the exception of the addition of 100

$437 \mu \mathrm{M}$ TCEP to the lysis buffer for NTSR1. Preparation of MOR/Mb6 complex was identical

438 to that of receptor Nb6 complex, with the omission of the HRV-3C cleavage to remove

439 eGFP as it was not present in the construct.

\section{Cryo-EM Sample Preparation}

442 All samples were prepared on glow-discharged holey gold grids (Quantifoil ultrAufoil

$443 \mathrm{R} 1.2 / 1.3$ ), blotted in a FEI Vitrobot Mark IV (Thermo Fisher Scientific) at $4^{\circ} \mathrm{C}$ and $100 \%$

444 humidity, and plunge frozen into liquid ethane. Blotting conditions for each sample were

445 as follows: $3.0 \mu$ of NTSR1/Nb6 complex at $16 \mathrm{mg} / \mathrm{ml}$ with an additional $0.05 \%$ beta OG;

$4463.0 \mu \mathrm{l}$ of MOR/Mb6 complex at $5 \mathrm{mg} / \mathrm{ml}$; and $2.5 \mu \mathrm{l}$ of SSTR2/Nb6 complex at $16 \mathrm{mg} / \mathrm{ml}$

447 with an additional $0.05 \%$ beta OG.

\section{Cryo-EM Data Collection}


450 All samples were collected on a KRIOS electron microscope at an accelerating voltage

451 of $300 \mathrm{kV}$, with an energy filter for NTSR1/Nb6 and MOR/Mb6 and without an energy filter

452 for SSTR2/Nb6. All data was collected through SerialEM with beam tilt compensation and

453 recorded on a Gatan K3 direct electron detector. The resulting image stacks have a pixel

454 size of $0.434 \AA$ for NTSR1/Nb6 and MOR/Mb6 and $0.426 \AA$ for SSTR2/Nb6, all in super

455 resolution mode. Each $\mathrm{NTSR} 1 / \mathrm{Nb6}$ image stack is composed of 71 frames with an

456 incident electron dose of $0.85 \mathrm{e}^{-} / \AA^{2}$ per frame, for a total dose of $61 \mathrm{e}^{-} / \AA^{2} / \mathrm{s}$ per micrograph.

457 Each MOR/Mb6 image stack is composed of 63 frames with an incident electron dose of

$4580.98 \mathrm{e}^{-/} / \AA^{2}$ per frame, for a total dose of $61 \mathrm{e}^{-/} \AA^{2} / \mathrm{s}$ per micrograph. Each SSTR2/Nb6

459 image stack is composed of 55 frames with an incident electron dose of $1.26 \mathrm{e}^{-} / \AA^{2}$ per

460 frame, for a total dose of $69 \mathrm{e}^{-/ \AA^{2}} / \mathrm{s}$ per micrograph.

\section{Cryo-EM Data Processing}

463 A pictorial depiction of each cryoEM processing workflow can be found in Extended Data

464 Figs. 2c, 4c, and 6c. Briefly, dose-fractionated image stacks were imported into RELION-

$4653.1^{31}$ and subjected to beam-induced motion correction and dose weighting with

466 MotionCor2 ${ }^{32}$. Contrast transfer function parameter estimation was performed with

467 CTFFIND-4.133. Particle selection and extraction was performed with the Laplacian

468 autopicker function of RELION-3.1 on micrographs with a CTF fit better than $3.5 \AA$. The

469 extracted particle stack was imported into CryoSPARC ${ }^{34}$ and subjected to multiple rounds

470 of 2D classification followed by iterative rounds of ab initio reconstruction with multiple

471 classes and heterogeneous refinement to further classify particles. In early 3D iterations,

472 particles from poor 3D classes were subjected to additional 2D classification and good 
473 particles were 'rescued' for further 3D classification. Once iterative ab initio and

474 heterogenous refinement no longer produced higher resolution reconstruction, particles

475 were re-imported to RELION-3.1. In the cases of hNTSR1 and MOR CTF fit cutoffs of 3.1

$476 \AA$ and $3.3 \AA$ were applied, respectively. Particles were then subjected to 3D refinement in

477 RELION-3.1 followed by Bayesian polishing. Polished particles were then imported back

478 to CryoSPARC for non-uniform refinement, global CTF refinement based on optics group,

479 and repeated non-uniform and local refinement.

\section{Model Building and Refinement}

482 All initial models were rigid body fit into cryo-EM maps using the ChimeraX ${ }^{35}$ software.

483 For hNTSR1, the cryo-EM model of active state hNTSR1 was used as the initial model

484 (PDB:6OS919); for MOR the crystal structure of inactive MOR bound to a covalent

485 antagonist was used as the initial model (PDB:4DKL ${ }^{36}$ ), and for SSTR2 a homology model

486 generated from KOR (PDB:6VI4 ${ }^{4}$ ) was used. In all cases KOR/Nb6 complex (PDB:6VI4 $\left.{ }^{4}\right)$

487 was aligned to the receptor to produce the initial model for the nanobody. All structures

488 were manually refined in $\mathrm{COOT}^{37}$ with iterative real-space refinement in Phenix ${ }^{38}$. Once

489 accurate modeling was achieved for the protein components, the GemSpot pipeline ${ }^{39}$ was

490 used for automatic modeling of the ligands alvimopan and SR48692 into MOR and

491 hNTSR1 respectively. Final refinement was executed in Phenix, followed by model-free

492 Phenix map modification for NTSR1 and MOR ${ }^{40}$. All maps displayed in this work are those

493 prior to model-free map modification unless stated otherwise.

\section{Molecular Dynamics Simulations}


496 Simulations of the KOR/Nb6 complex started with the crystal structure PDB:6VI4, with the

497 pose of JDTic replaced with that of PDB:4DJH ${ }^{21}$. Maestro's protein preparation tool was

498 used to assign protonation states, optimize hydrogen bonding, and build missing

499 sidechains and loops. Once the receptor was prepared, the orientation of proteins in

500 membranes webserver (OPM) $)^{41}$ was used to align the receptor as it would be in a

501 membrane in the xy plane and the CHARMM-GUI ${ }^{42}$ was used to build the full system with

502 a 1-palmitoyl-2-oleoyl-sn-glycero-3-phosphocholine POPC/CHS lipid bilayer, TIP3P

503 water ${ }^{43}$, and $150 \mathrm{mM} \mathrm{NaCl}$. PSF and PDB files were generated with the OPLS-AA/M ${ }^{44}$

504 forcefield in $\mathrm{VMD}^{45}$ for the system. For Mb6, a homology model was built from PDB:6XVI

505 with the Prime homology modeling tool ${ }^{46}$. This model was solvated in TIP3P water and

$506150 \mathrm{mM} \mathrm{NaCl}$ in VMD for simulation.

$507 \quad$ Molecular dynamics simulations were carried out in NAMD $^{47}$ with a 2 fs timestep

508 with SHAKE and SETTLE algorithms ${ }^{48,49}$, with a Langevin thermostat and a Nosé-Hoover

509 Langevin piston barostat at 1 atm with a period of 50 fs and decay of 25 fs. Periodic

510 boundary conditions were used with nonbonded interactions smoothed starting at $10 \AA$ to

$51112 \AA$ with long-range interactions treated with particle mesh Ewald (PME). The system

512 was minimized for 1,500 steps and then slowly heated from 0 to $303.15 \mathrm{~K}$ in increments

513 of $20 \mathrm{~K}$ simulating for 0.4 ns at each increment. For the Kappa/Nb6 simulations, all non-

514 hydrogen, non-water, and non-ion atoms were restrained with a $1 \mathrm{kcal} / \mathrm{mol} / \AA^{2}$ harmonic

515 restraint during heating and an additional $10 \mathrm{~ns}$ of equilibration. $1 \mathrm{kcal} / \mathrm{mol} / \AA^{2}$ harmonic

516 restraints were used for an additional $10 \mathrm{~ns}$ of equilibration on all non-hydrogen protein

517 atoms followed by a final $10 \mathrm{~ns}$ of restrained equilibration with $1 \mathrm{kcal} / \mathrm{mol} / \AA^{2}$ harmonic

518 restraint on only $\mathrm{C} \alpha$ atoms. The first 30 ns of unrestrained molecular dynamics were also 
519 considered to be equilibration, with an additional 500 ns of simulation performed. All

520 simulations were performed in triplicate. For Mb6, the first 30 ns of unrestrained molecular

521 dynamics were treated as equilibration, and 100 ns of triplicate molecular dynamics

522 simulations were performed.

\section{Docking}

524 The cryo-EM structure of active MOR (PDB:6DDE) ${ }^{3}$ was prepared in Maestro and 525 carfentanil was docked with Glide XP docking ${ }^{50}$.

526

\section{References}

528 1. Robertson, M. J., Meyerowitz, J. G. \& Skiniotis, G. Drug discovery in the era of cryo529 electron microscopy. Trends in Biochemical Sciences (2021). 530 doi:10.1016/j.tibs.2021.06.008

531 2. Hauser, A. S., Attwood, M. M., Rask-Andersen, M., Schiöth, H. B. \& Gloriam, D. E. Trends 532 in GPCR drug discovery: New agents, targets and indications. Nat. Rev. Drug Discov. $533 \quad$ (2017). doi:10.1038/nrd.2017.178

534 3. Koehl, A. et al. Structure of the $\mu$-opioid receptor-Gi protein complex. Nature 558, 547-552 $535 \quad$ (2018).

536 4. Che, T. et al. Nanobody-enabled monitoring of kappa opioid receptor states. Nat. Commun. 537 (2020). doi:10.1038/s41467-020-14889-7

538 5. Gully, D. et al. Biochemical and pharmacological profile of a potent and selective 539 nonpeptide antagonist of the neurotensin receptor. Proc. Natl. Acad. Sci. U. S. A. (1993). 540 doi:10.1073/pnas.90.1.65

541 6. Uchański, T. et al. Megabodies expand the nanobody toolkit for protein structure 542 determination by single-particle cryo-EM. Nat. Methods (2021). doi:10.1038/s41592-020$543 \quad 01001-6$ 
544 7. Zimmerman, D. M. et al. Discovery of a Potent, Peripherally Selective trans-3,4-Dimethyl545 4-(3-hydroxyphenyl)piperidine Opioid Antagonist for the Treatment of Gastrointestinal $546 \quad$ Motility Disorders. J. Med. Chem. (1994). doi:10.1021/jm00041a003

547 8. Carraway, R. \& Leeman, S. E. Characterization of radioimmunoassayable neurotensin in 548 the rat. Its differential distribution in the central nervous system, small intestine, and 549 stomach. J. Biol. Chem. (1976). doi:10.1016/s0021-9258(17)32938-1

550 9. Christou, N. et al. Neurotensin pathway in digestive cancers and clinical applications: an overview. Cell Death and Disease (2020). doi:10.1038/s41419-020-03245-8

552 10. Liu, J. et al. neurotensin receptor 1 antagonist SR48692 improves response to carboplatin by enhancing apoptosis and inhibiting drug efflux in ovarian cancer. Clin. Cancer Res. (2017). doi:10.1158/1078-0432.CCR-17-0861

555 11. Volkow, N. D. \& Blanco, C. The changing opioid crisis: development, challenges and opportunities. Molecular Psychiatry (2021). doi:10.1038/s41380-020-0661-4

557 12. Volkow, N. D. \& Collins, F. S. The Role of Science in Addressing the Opioid Crisis. N. Engl. J. Med. (2017). doi:10.1056/nejmsr1706626

559 13. Günther, T. et al. International union of basic and clinical pharmacology. CV. somatostatin receptors: Structure, function, ligands, and new nomenclature. Pharmacol. Rev. (2018). doi:10.1124/pr.117.015388

562 14. Ballesteros, J. A. \& Weinstein, H. Integrated methods for the construction of threedimensional models and computational probing of structure-function relations in G proteincoupled receptors. Methods Neurosci. (1995). doi:10.1016/S1043-9471(05)80049-7

565 15. Stoeber, M. et al. A Genetically Encoded Biosensor Reveals Location Bias of Opioid Drug Action. Neuron (2018). doi:10.1016/j.neuron.2018.04.021

567 16. Deluigi, M. et al. Complexes of the neurotensin receptor 1 with small-molecule ligands 568 reveal structural determinants of full, partial, and inverse agonism. Sci. Adv. (2021). 569 doi:10.1126/sciadv.abe5504 
570 17. Mittl, P. R., Ernst, P. \& Plückthun, A. Chaperone-assisted structure elucidation with 571 DARPins. Current Opinion in Structural Biology (2020). doi:10.1016/j.sbi.2019.12.009

572 18. Scott, D. J., Kummer, L., Egloff, P., Bathgate, R. A. D. \& Plückthun, A. Improving the apo573 state detergent stability of NTS1 with CHESS for pharmacological and structural studies. 574 Biochim. Biophys. Acta - Biomembr. (2014). doi:10.1016/j.bbamem.2014.07.015

575 19. Kato, H. E. et al. Conformational transitions of a neurotensin receptor 1-Gi1 complex. $576 \quad$ Nature 572, 80-85 (2019).

577 20. Russo, C. J. \& Passmore, L. A. Ultrastable gold substrates for electron cryomicroscopy. 578 Science (80-. ). (2014). doi:10.1126/science.1259530

579 21. Wu, H. et al. Structure of the human K-opioid receptor in complex with JDTic. Nature (2012). doi:10.1038/nature10939

581 22. Van Bever, W. F. M., Niemegeers, C. J. E. \& Schellekens, K. H. L. P. A. J. N 4 substituted 1 (2 arylethyl) 4 piperidinyl $\mathrm{N}$ phenylpropanamides, a novel series of extremely potent analgesics with unusually high safety margin. Arzneimittel-Forschung/Drug Res. (1976).

584 23. Robertson, M. J., Meyerowitz, J. M., Panova, O., Borrelli, K. W., Skiniotis, G. Plasticity in Ligand Recognition at Somatostatin Receptors. (2021)

586 24. Tunyasuvunakool, K. et al. Highly accurate protein structure prediction for the human proteome. Nature (2021). doi:10.1038/s41586-021-03828-1

588 25. Baek, M. et al. Accurate prediction of protein structures and interactions using a three-track neural network. Science (80-. ). (2021). doi:10.1126/science.abj8754

590 26. Kong, H., Raynor, K., Yasuda, K., Bell, G. I. \& Reisine, T. Mutation of an aspartate at 591 residue 89 in somatostatin receptor subtype 2 prevents $\mathrm{Na}+$ regulation of agonist binding 592 but does not alter receptor-G protein association. Mol. Pharmacol. (1993).

593 27. Martin, S., Botto, J. M., Vincent, J. P. \& Mazella, J. Pivotal role of an aspartate residue in 594 sodium sensitivity and coupling to G proteins of neurotensin receptors. Mol. Pharmacol. $595 \quad$ (1999). doi:10.1124/mol.55.2.210 
596 28. Yabaluri, N. \& Medzihradsky, F. Regulation of $\mu$-opioid receptor in neural cells by 597 extracellular sodium. J. Neurochem. (1997). doi:10.1046/j.1471-4159.1997.68031053.x

598 29. Fenalti, G. DRUG DISCOVERY Molecular control of delta -opioid receptor signalling. Nat. $599 \quad-L O N D O N-(2014)$.

600 30. Towns, J. et al. XSEDE: Accelerating scientific discovery. Comput. Sci. Eng. (2014). $601 \quad$ doi:10.1109/MCSE.2014.80

602 31. Zivanov, J. et al. RELION-3: New tools for automated high-resolution cryo-EM structure 603 determination. bioRxiv (2018). doi:10.1101/421123

604 32. Zheng, S. Q. et al. MotionCor2: Anisotropic correction of beam-induced motion for 605 improved cryo-electron microscopy. Nature Methods 14, 331-332 (2017).

606 33. Rohou, A. \& Grigorieff, N. CTFFIND4: Fast and accurate defocus estimation from electron micrographs. J. Struct. Biol. 192, 216-221 (2015).

608 34. Punjani, A., Rubinstein, J. L., Fleet, D. J. \& Brubaker, M. A. CryoSPARC: Algorithms for 609 rapid unsupervised cryo-EM structure determination. Nat. Methods (2017). doi:10.1038/nmeth.4169

611 35. Pettersen, E. F. et al. UCSF ChimeraX: Structure visualization for researchers, educators, and developers. Protein Sci. (2021). doi:10.1002/pro.3943

613 36. Manglik, A. et al. Crystal structure of the $\mu$-opioid receptor bound to a morphinan 614 antagonist. Nature (2012). doi:10.1038/nature10954

615 37. Emsley, P., Lohkamp, B., Scott, W. G. \& Cowtan, K. Features and development of Coot. Acta Crystallogr. Sect. D Biol. Crystallogr. 66, 486-501 (2010).

617 38. Adams, P. D. et al. PHENIX: A comprehensive Python-based system for macromolecular 618 structure solution. Acta Crystallogr. Sect. D Biol. Crystallogr. 66, 213-221 (2010).

619 39. Robertson, M. J., van Zundert, G. C. P., Borrelli, K. \& Skiniotis, G. GemSpot: A Pipeline for 620 Robust Modeling of Ligands into Cryo-EM Maps. Structure (2020). 621 doi:10.1016/j.str.2020.04.018 
622 40. Terwilliger, T. C., Ludtke, S. J., Read, R. J., Adams, P. D. \& Afonine, P. V. Improvement of 623 cryo-EM maps by density modification. Nat. Methods (2020). doi:10.1038/s41592-020$624 \quad 0914-9$

625 41. Lomize, M. A., Pogozheva, I. D., Joo, H., Mosberg, H. I. \& Lomize, A. L. OPM database 626 and PPM web server: Resources for positioning of proteins in membranes. Nucleic Acids 627 Res. 40, (2012).

628 42. Lee, J. et al. CHARMM-GUI Input Generator for NAMD, GROMACS, AMBER, OpenMM, 629 and CHARMM/OpenMM Simulations Using the CHARMM36 Additive Force Field. J. 630 Chem. Theory Comput. 12, 405-413 (2016).

631 43. Jorgensen, W. L., Chandrasekhar, J., Madura, J. D., Impey, R. W. \& Klein, M. L. 632 Comparison of simple potential functions for simulating liquid water. J. Chem. Phys. 79, $633926-935(1983)$ 9

634 44. Robertson, M. J., Tirado-Rives, J. \& Jorgensen, W. L. Improved Peptide and Protein 635 Torsional Energetics with the OPLS-AA Force Field. J. Chem. Theory Comput. 11, 3499$6363509(2015)$.

637 45. Humphrey, W., Dalke, A. \& Schulten, K. VMD: Visual molecular dynamics. J. Mol. Graph. $638 \quad 14,33-38(1996)$.

639 46. Jacobson, M. P. et al. A Hierarchical Approach to All-Atom Protein Loop Prediction. Proteins Struct. Funct. Genet. (2004). doi:10.1002/prot.10613

641 47. Phillips, J. C. et al. Scalable molecular dynamics with NAMD. Journal of Computational Chemistry 26, 1781-1802 (2005).

643 48. Ryckaert, J. P., Ciccotti, G. \& Berendsen, H. J. C. Numerical integration of the cartesian 644 equations of motion of a system with constraints: molecular dynamics of n-alkanes. J. $645 \quad$ Comput. Phys. (1977). doi:10.1016/0021-9991(77)90098-5

646 49. Miyamoto, S. \& Kollman, P. A. Settle: An analytical version of the SHAKE and RATTLE 647 algorithm for rigid water models. J. Comput. Chem. (1992). doi:10.1002/jcc.540130805 
bioRxiv preprint doi: https://doi.org/10.1101/2021.11.02 466983. this version posted November 4, 2021. The copyright holder for this preprint

(which was not certified by peer review) is the author/funder, who has granted bioRxiv a license to display the preprint in perpetuity. It is made available under aCC-BY-NC-ND 4.0 International license.

648 50. Friesner, R. A. et al. Extra precision glide: Docking and scoring incorporating a model of

649 hydrophobic enclosure for protein-ligand complexes. J. Med. Chem. 49, 6177-6196

$650 \quad$ (2006).

651 